\title{
For Better or For Worse: academic partnerships for data services
}

\author{
by Diane Geraci ${ }^{I}$ \\ Binghamton University \\ State University of New York
}

\section{Introduction}

While there is no one model for providing services for data in colleges and universities, it is increasingly common for various constituencies to cooperate, especially in lean fiscal years. There are both positive and negative aspects to pooling resources in such a "marriage of convenience." Although not the solution for everyone, this paper will take a look at a partnership among two academic departments, Computing Services, the Libraries, and the Provost's office at Binghamton University, State University of New York. 'It will suggest advantages and disadvantages for those considering cooperative ventures at their institutions.

\section{From this day forward, for better for worse, for richer for poorer, in sickness and in health, to love and to cherish, till death us do part ...}

Data library and service operations in academic institutions in North America have in many instances seen a reduction in resources in the last five years. In some cases, this has threatened the existence of some or even all services. In others, it has caused data professionals and administrators to be creative and forge new arrangements to maintain or even enhance basic levels of service for their clientele. Because data service organizations vary considerably from academic institution to institution, there is no single or simple way to diagram a preferred organizational structure for data service. What works in one academic setting, may not in another. Services seen as basic at one university may be on a wish list at others. Size and diversity of user groups also vary depending on programmatic and research agendas. In any case, optimal staffing and funding levels are directly related to the level of service needed by an institution's primary clientele. Unfortunately, even minimal resource levels may not be possible at some institutions.

Pooling resources among departments and units across a college or university can be an option where a separately funded "data center" or "data library" does not exist, or, when an existing service is faced with dissolution. While these "marriages of convenience not suitable for all organizations, there are significant advantages and disadvantages of academic partnerships. They are especially worth exploring if an institution faces "rightsizing" or consolidating services. These partnerships rely on the ability of various constituencies to work together, an agreed upon common purpose, mutual respect, and tolerance.

\section{from this day forward...}

An institution's history of providing quantitative, social research support on a given campus will often set the stage for future service configurations. Because of this it can be difficult to change support paradigms, although it is certainly possible and even necessary in some cases.

At Binghamton University, State University of New York, the Political Science Department in conjunction with an organized research center, provided support for quantitative social data for two decades. In 1990, a time of considerable fiscal uncertainly in the University system, the impending closing of that research center necessitated rethinking the way in which we were organized to provide data services. For the most part this meant fulfilling our Inter-university Consortium for Political and Social Research (ICPSR) membership responsibilities and related data services.

After a series of extensive consultations with administrators, faculty and staff, the Libraries agreed to assume responsibility for 'data services.' This primarily entailed maintaining formal relationships with ICPSR and later the U.S. Bureau of the Census' State Data Center Program. Ultimately this meant that the Libraries would:

. maintain formal relations with ICPSR

.serve as liaison for the State Data Center Program

. assume fiscal responsibility for ICPSR membership after an initial transfer of monies from the Provost's office

- provide customer services, particularly identifying and ordering data 
.collect and maintain codebooks, related technical documentation and statistical manuals

.provide user consultations, research assistance and referrals .cooperate with Academic Computing, to make data available and to provide complementary services

.cooperate with the Economics and Political Science departments, and the Assistant Provost for Graduate Studies and Teaching to assign two ICPSR/Data Services graduate assistants to the Libraries.

The formal change in service occurred in July 11991 to coincide with the new fiscal year. However, Academic Computing, the Libraries, the Political Science department, and the organized research center had already begun the process of working together several years before. This early period effectively served as a 'getting to know you" phase where each unit's service orientation and working patterns became known. Evolving service plans and position descriptions assisted in making clear who would be responsible for which aspect of the reconstituted service.

for better for worse...

Commitment of each constituency is essential for a service that exists through the shared agreement of its partners. The best strategy for success is creating a winwin situation whereby each of the partners benefits from contributing to the service. A benefit may mean better meeting the mission of the unit, such as a library or computing service that serves the entire academic community. From an institutional point of view it may mean reducing duplicate purchases or services. It certainly will mean providing the kind of research support desired by relevant academic programs. It can also mean acknowledging that going it alone might not provide the depth and range of services needed.

While good will and intentions may characterize a shared agreement to provide service, a written plan is well worth the effort. Support staff and administrators do change. A written service plan cannot absolutely guarantee the continued cooperation of each unit but it does provide a framework and codification of responsibilities.

After seven years of sharing responsibility for data services on the Binghamton campus, several benefits are evident. They include:

- $\quad$ ICPSR membership benefits are more widely available to all constituencies on campus. There had been a perception that everyone knew about the ICPSR and the extent of their data holdings. This turned out not to the case. New faculty and graduate students continually arrive on campus and existing campus instructors and researchers have new data needs. Researchers in departments not traditionally employing quantitative research methodologies may begin doing so. There is a continual need for dissemination of information about new data and related data news. For example, only one department knew about the ICPSR Summer Program in Quantitative Methods before the Libraries coordinated the membership services.

- Duplication of data acquisitions was reduced. Because data support originally resided in the school of arts and sciences, other schools and divisions often bought there own data directly from producers. We found that much of these data were available via our ICPSR membership. This was especially true for health data and economic time series data.

- Integration of data collected in several media is a positive by-product of centering access to data in the Libraries. Print resources, CD-ROMS, diskettes, remote access via the Internet, and commercial services already are available in or through the Libraries. Making the Libraries the first stop to ascertain if data are available on mainframe cartridge tape has brought together conceptually if not physically, access to related resources.

- Existing expertise is utilized; that is, information management skills, computing skills and service orientation in the Libraries; technical, computing and statistical skills from Computer Services; research skills of the departmental graduate assistants.

- Skills shared across units increase the skills of all contributors to the service. Graduate students particularly gain solid experience working with data and valuable statistical programming skills.

- Cooperation with other units on campus increases awareness of research needs as well as understanding of different campus cultures. Daily contact with colleagues in other campus units greatly fosters understanding and respect for each other's work.

Several difficulties or less positive aspects of the partnership also became apparent.

We also found:

- ICPSR resources became more widely used on campus making it difficult for part-time staff in the several units providing support to keep up with demand. Statistics showed a substantial increase in data use on our campus as a result of the reconstituted 
service. Staff in the Libraries and in Academic Computing found that an increased percentage of their work week supported data services. Some reorganization of duties occurred in each unit with the pressure being born by existing staff members. Similarly, the service began with one graduate assistant. It soon became clear that one was insufficient and we were able to negotiate for another student.

- Reliance on graduate student support entails constant training and rotation of staff. Considerable fluctuations in the quality of service regularly occur.

- Additional permanent staff is desirable, but thus far, has been unattainable. Research level support is very time-consuming. Permanent staff and new lines are difficult to acquire. They would assist in providing consistent service and allow for performance of needed tasks, especially as the number of users increases and users' request an increased level of service.

- Keeping current with data services developments requires additional space and equipment. Changes in computing platforms and storage devices require new hardware and software. Decisions made in one unit may affect another. For example, the decision by Computing Services to stop maintenance of 9-track tape drives has consequences for the way that the Libraries order data.

- New skills are required. For example, knowledge of database maintenance, cataloging, or statistical programming, and understanding research design may be necessary for data services staff to provide certain services. For already overextended staff, there is not adequate time for learning new processes or acquiring necessary skills. The aptitudes of existing staff for acquiring new skills will also vary.

- Cooperating with other units on campus is difficult in practice. Conflicting priorities in a unit or between units may be difficult to resolve. Politics internal to a unit are less easily negotiated by those outside the unit. Service orientations or philosophies of the partners may differ.

in sickness and in poor-health...

In times of staff reduction, fiscal uncertainty, competing demands in a unit, or simply a reprioritization of needs or goals, a joint service can suffer the consequences. There can be real concerns for the integrity of the service as a whole if a key group withdraws its support. When individual units experience shifting priorities or staff reductions the danger exists that the shared service will fall to the bottom of the list of things to do, or worse, will no longer be supported. When there are administrative changes the partners in the service may need to renew their "vows."

While living with a small degree of uncertainty is admissible, a crisis can arise if one contributor to the service can no longer participate or even temporarily suspends participation. Major disruption of service or stress on the other partners can occur if one unit is unable to meet their obligations.

There is not a way to absolutely ensure that no change will occur in a partner's commitment to the relationship. There are ways, though, to engender support for the service and keep it on the priority list of each partner. Relying on a core group of researchers as an "advisory group" is one way to get feedback from users.

Measuring the amount of data ordered, number of users assisted, computer usage, and any other relevant factor at an institution can demonstrate the utility and necessity of the data service to administrators.

to love and to cherish...

When there is stability in the service and researchers' needs are being met, all partners deserve congratulations for cooperating across units and effectively working together to create a viable service. This is the "feel good" outcome of a win-win situation and should be enjoyed. Lest complacency cause problems, it is a good idea to reaffirm what works with the arrangement and what can be handled in a better way. Assessment

during the good times is much less threatening then when the sky is falling due to impending budget cuts or some other "natural" academic disaster. Several methods work well to evaluate the service including meeting with the primary front line staff in each unit, consulting an advisory group of researchers, and surveying past and prospective users of the service. Taking the time for assessment is a positive way to renew the agreement and service plan(s) of the units involved and make any necessary adjustments.

till death us do part?

Binghamton's "marriage of convenience" came at time when data support on the campus was in jeopardy. It has served the university community well in its time. It does not mean that this is the only way to provide data services or that another type of service will not evolve from it.

There are several reasons a partnership such as Binghamton's might cease to continue:

. The service is no longer necessary. There may be 
other ways to meet the need of data users. Schools or departments might decide to provide some of their own services. National or international consortia and computer networks may provide more data services negating the need for some local services. It is difficult to imagine, though, that some measure of local support will not be necessary, even in a future of distributed services over "the net." There certainly will be a time when the service needs to be reformulated or reconstituted.

. One or more of the partners cannot afford the commitment of staff and/or resources. A worst case scenario is the service dies. Another possibility is that the other partners are able to pick up the slack. In the case where the partners are unable to absorb additional responsibilities, providing a reduced level of service may be necessary.

. Cooperation is no longer possible between the partners. One of more of the partners may experience a change in their mission, unresolvable disagreements may occur between partners, or administrative prerogative may preclude further cooperation.

Providing data services through an academic partnership can be very rewarding. Forging key relationships between disparate units and seeing positive results in support of research and teaching are successful outcomes. Before embarking on a cooperative venture, careful consideration of a partnership model's suitability for the needs and culture of an institution is necessary.

1. Paper presented at IASSIST 1994 in San Francisco. 